\title{
INVENTORY OF SCARABAEID BEETLES (COLEOPTERA) FROM MADHYA PRADESH, INDIA
}

\author{
Kailash Chandra
}

Zoological Survey of India, Central Regional Station, Jabalpur, Madhya Pradesh 482002, India.

\begin{abstract}
Scarabeid beetles (Coleoptera) were studied in Madhya Pradesh as part of an extensive study of biodiversity in the State by the Zoological Survey of India. This paper presents a checklist of scarabeid beetles from Madhya Pradesh, which total up to 94 taxa belonging to 9 subfamilies.
\end{abstract}

\section{Keywords}

Scarabeid beetles, coleoptera, Madhya Pradesh, distribution, checklist

\section{Introduction}

The scarabaeid beetles of the order Coleoptera include both useful as well as harmful insects. The coprophagous beetles generally known as dung beetles, play an important role in nature's sanitation by feeding on the dung. The phytophagous beetles commonly known as chafers are pests of agricultural crops, plantations and forests.

The family Scarabaeidae is the largest family of insects, which contains more than 30,000 species in the world (Fincher, 1981). The first comprehensive account of scarabaeid beetles of the Indian region was published by Arrow $(1910,1917,1931)$ in three volumes of Fauna of British India, where in 58 species were reported from Madhya Pradesh, which was earlier part of Central India. Balthasar (1963a, 1963b, 1964) wrote monographs on the two subfamilies Scarabaeinae and Aphodiinae of Palaearctic and Oriental regions. Newton and Malcolm (1985) studied the dung beetles of wild mammals in Kanha Tiger Reserve and 22 species were recorded, of which 13 were identified up to species level. While working on the faunal diversity of Pachmarhi Biosphere Reserve (Hoshangabad), Chandra and Singh (communicated) recorded 18 species of scarabaeid beetles belonging to 11 genera under 6 subfamilies, which are new additions to the fauna of Hoshangabad.

Received on 22 July 2000 Accepted on 29 August 2000
Since comprehensive studies on insect diversity of Madhya Pradesh is lacking completely, the Zoological Survey of India, Central Regional Station, Jabalpur undertook detailed studies on all the groups of insects. This would not only help in the inventorisation of the faunal diversity of Madhya Pradesh but also in formulating management strategy for the conservation of biodiversity. In the first attempt, the scarabaeid beetles collected through extensive surveys of Madhya Pradesh by the Zoological Survey of India were worked out, which revealed the presence of 94 species belonging to 30 genera distributed under nine subfamilies (Table 1). Of these, six species viz. Orphnus parvus (Wied.), Chiron cylindrus (Fabricius), Chironitis arrowi Janssens, Heliocopris gigas (Linnaeus), Oxyadoretus nasutus Arrow and Protaetia alboguttata Vigors are reported for the first time from Madhya Pradesh.

\section{Acknowledgements}

The author is grateful to Dr. J.R.B. Alfred, Director, Zoological Survey of India for providing all the necessary facilities and encouragement; to Shri. K. Vinod, Stenographer, for typing the manuscript; and to Shri. M.L. Dhimar, Junior Zoological Assistant for setting and pinning of the beetles for the present studies.

\section{References}

Arrow, G.J. (1910). The Fauna of British India including Ceylon and Burma, Col. Lamell. I (Cetoniinae and Dynastinae), Taylor \& Francis, London : V-XIV, 322 pp., 76 figs., 2 pls.

Arrow, G.J. (1917). The Fauna of British India including Ceylon and Burma, Col. Lamell. II (Rutelinae, Desmonychinae and Euchiirinae). Taylor \& Francis, London : V-XIII, 387 pp., 77 figs., 5 pls.

Arrow, G.J. (1931). The Fauna of British India including Ceylon and Burma, Col. Lamell. III (Coprinae), Taylor \& Francis, London : V-XII, 428 pp., 61 figs., 19 pls., map.

Balthasar, V. (1963a). Monographie der Scarabaeidae und Aphodiidae der Palaearktischen und Orientalischen Region. Pragus, Verl. Tschechosl. Akad. Wissensch. I, 391 pp., 137 figs., 24 pls.

Balthasar, V. (1963b). Monographie der Scarabaeidae und Aphodiidae der Palaearktischen und Orientalischen Region. Prague, Verl. Tschechosl. Akad. Wissensch II, 627 pp., 226 figs., 16 pls.

Balthasar, V. (1964). Monographie der Scarabaeidae und Aphodiidae der Palaearktischen und Orientalischen Region. Prague, Verl. Tschechosl. Akad. Wissensch. III, pp. 652. 224 figs. 


\begin{tabular}{|c|c|c|c|c|c|}
\hline Scientific Name & Distribution in M.P. & Reference & Scientific Name & Distribution in M.P. & Reference \\
\hline \multicolumn{5}{|l|}{ Subfamily: Hybosorinae } & Chandra \& Singh, comm. \\
\hline 1 Hybosorus illigeri Reiche & M.P. & Kuijten, 1983 & 26 Gymnopleurus dejeani Castelnau & Indore & Arrow, 1931 \\
\hline \multirow[t]{3}{*}{2 Hybosorus orientalis (Westwood) } & \multirow[t]{3}{*}{ Jabalpur } & Kuijten, 1983, & 27 Gymnopleurus gemmatus Harold & Jabalpur & Arrow, 1931 \\
\hline & & \multirow[t]{2}{*}{ Chandra \& Singh, comm. } & 28 Gymnopleurus maculosus Macleay & M.P. & Arrow, 1931 \\
\hline & & & 29 Gymnopleurus miliaris (Fabricius) & Hoshangabad, Jabalpur & $\begin{array}{l}\text { Arrow, } 1931 \text {; Chandra \& } \\
\text { Singh. comm. }\end{array}$ \\
\hline \multirow[t]{2}{*}{3 Orphnus parvus (Wied.) $)^{*}$} & \multirow{3}{*}{ Jabalpur } & \multirow{3}{*}{ JSB on 3.vii.1964 } & 30 Gymnopleurus mundus (Wieddman) & Hoshangabad, Mandla & Newton \& Malcolm, 1985; \\
\hline & & & $(=$ Garreta mundus $)$ & & Chandra \& Singh, comm. \\
\hline$\underline{\text { Subfamily: Chironinae }}$ & & & 31 Gymnopleurus opacus (Redt.) & Hoshangabad & Chandra \& Singh, comm. \\
\hline 4 Chiron cylindrus (Fabricius) ${ }^{*}$ & \multirow[t]{2}{*}{ Jabalpur } & \multirow[t]{2}{*}{$\begin{array}{l}\text { JSB, MLK on 6.vii.1964; } \\
\text { 19, 21.viii.1964 }\end{array}$} & $\begin{array}{l}32 \text { Gymnopleurus parvus (Macl.) } \\
33 \text { Gymnopleurus sinuatus (Olivier) }\end{array}$ & Hoshangabad & Chandra \& Singh, comm. \\
\hline Subfamily: Aphodiinae & & & (= Progymnopleurus sinuatus Olivier) & Mandla & Newton \& Malcolm, 1985 \\
\hline 5 Aphodius moestus Fabricius & Jabalpur & Balthasar, 19 & 34 Gymnopleurus sumptuosus Castelnau & Indore & Arrow, 1931 \\
\hline 6 Aphodius (Loboparius) vitalisi Bouc. & M.P. & Balthasar, 1964 & 35 Heliocopris bucephalus (Fabricius) & Jabalpur, Mandla & Arrow, 1931 \\
\hline 7 Chaetopisthes (C.) fulvus Westw. & M.P. & Balthasar, 1964 & 36 Heliocopris gigas (Linnaeus) & Gwalior & RKS on 3.ix.1995 \\
\hline 8 Termitopisthes laticollis Wasm. & Hoshangabad & Balthasar, 1964 & 37 Liatongus rhadamistus (Fabricius) & Balaghat, Riwa, Umaria & Arrow, 1931 \\
\hline \multicolumn{2}{|c|}{9 Termitopisthesernutioustges wasmanni (A. Schmidt) Mandla } & \multirow[t]{2}{*}{ Balthasar, 1964} & 38 Oniticellus cinctus (Fabricius) & Hoshangabad, Riwa, Shahdol & $\begin{array}{l}\text { Arrow, } 1931 \\
\text { Chandra \& Singh, comm. }\end{array}$ \\
\hline Subfamily: Scarabaeinae & & & 39 Oniticellus spinipes Roth & Riwa, Shahdol, Umaria & Arrow, 1931 \\
\hline 10 Caccobius unicornis (Fab.) & \multirow{6}{*}{$\begin{array}{l}\text { Hoshangabad } \\
\text { Mandla, Jabalpur, } \\
\text { Hoshangabad } \\
\text { Jabalpur, Narsinghpur } \\
\text { Gwalior, Mandla, Jabalpur }\end{array}$} & \multirow{6}{*}{$\begin{array}{l}\text { Arrow, } 1931 \\
\text { Newton \& Malcolm 1985; } \\
\text { Chandra \& Singh, comm. } \\
\text { Newton \& Malcolm, } 1985 \\
\text { Arrow, } 1931\end{array}$} & 40 Onitis brahma Lansberg & Betul, Balaghat & Arrow, 1931, Balthsar, \\
\hline \multirow[t]{2}{*}{11 Catharsius pithecius (Fabricius) } & & & & & $1963 \mathrm{~b}$ \\
\hline & & & 41 Onitis castaneus Redtenbacker & M.P. & Balthasar, 1963b \\
\hline 12 Catharsius molossus (Linnaeus) & & & 42 Onitis crassus Sharp & Jabalpur & Arrow, 1931, Balthsar, \\
\hline 13 Catharsius sagax Queensland & & & & & $1963 \mathrm{~b}$ \\
\hline \multirow{2}{*}{$\begin{array}{l}14 \text { Chironitis arrowi Janssens } \\
\text { (= Chironitis indicus Lansberg) }\end{array}$} & & & 43 Onitis falcatus (Wulfen) & M.P. & Balthasar, 1963b \\
\hline & Jabalpur & H.K. on 13.x.1965 & 44 Onitis philemon Fabricius & Indore, Bastar, Hoshangabad & Arrow, 1931 \\
\hline \multirow{5}{*}{$\begin{array}{l}15 \text { Copris andrewesi Waterhouse } \\
16 \text { Copris carinicus Gillet } \\
17 \text { Copris davisoni Waterhouse } \\
18 \text { Copris imitans Felsche }\end{array}$} & \multicolumn{2}{|c|}{ Indore, Seoni, Mandla, Jabalpur ～Arrow, 1931} & 45 Onitis subopacus Arrow & Mandla, Balaghat, Riwa & Arrow, 1931; \\
\hline & Mandla & Newton \& Malcolm, 1985 & & & Newton \& Malcolm, 1985 \\
\hline & Mandla & Newton \& Malcolm, 1985 & 46 Onitis virens Lansberg & Balaghat, Bastar & Arrow, 1931; Balthasar, \\
\hline & Indore, Seoni, Riwa,Mandla, & Arrow, 1931; & & & $1963 b$ \\
\hline & Balaghat, Shahdol, Umaria & Balthasar, 1963a & 47 Onthophagus abreui Arrow & Betul, Seoni & Arrow, 1931 \\
\hline 19 Copris pedarioides Lansberg & M.P. & Balthasar, 1963a & 48 Onthophagus aguns Gill & Indore, Seoni & Arrow, 1931 \\
\hline \multirow[t]{2}{*}{20 Copris repertus Walker } & Indore, Mandla, & Arrow, 1931; & 49 Onthophagus bonasus (Fabricius) & Betul, Balaghat & Arrow, 1931 \\
\hline & Umaria, Bastar & Newton \& Malcolm, 1985 & 50 Onthophagus brevicollis Arrow & Balaghat, Raigarh & Arrow, 1931 \\
\hline \multirow[t]{2}{*}{21 Copris signatus Walker } & \multirow[t]{2}{*}{ M.P. } & \multirow{2}{*}{$\begin{array}{l}\text { Arrow, 1931, Balthasar, } \\
\text { 1963a }\end{array}$} & 51 Onthophagus catta Fabricius & Indore, Betul, Balaghat & Arrow, 1931 \\
\hline & & & 52 Onthophagus centricornis (Fabricius) & Balaghat, Raigarh & Arrow, 1931 \\
\hline 22 Copris surdus Arrow & M.P. & Arrow, 1931 & 53 Onthophagus cervus (Fabricius) & Balaghat, Riwa, Umaria & Arrow, 1931 \\
\hline \multirow[t]{2}{*}{23 Drepanocerus setosus (Wiedman) } & \multirow{2}{*}{$\begin{array}{l}\text { Riwa, Shahdol, Umaria, } \\
\text { Bastar }\end{array}$} & \multirow{2}{*}{$\begin{array}{l}\text { Arrow, 1931; Balthasar, } \\
\text { 1963b }\end{array}$} & 54 Onthophagus dama(Fabricius) & Balaghat & Arrow, 1931 \\
\hline & & & 55 Onthophagus fasciatus Boucomont & Riwa, Shahdol, Umaria & Arrow, 1931 \\
\hline 24 Drepanocerus sinicus Harold & M.P. & Arrow, 1931; Balthasar, & 56 Onthophagus furcilliferBates & Bilaspur & Arrow, 1931 \\
\hline & & $1963 b$ & 57 Onthophagus griseosetosus Arrow & Mandla, Balaghat, Raigarh & Arrow, 1931; \\
\hline 25 Gymnopleurus cyaneus (Fabricius) & Hoshangabad, Mandla & Arrow, 1931; & & & Newton \& Malcolm, 1985 \\
\hline & & Newton \& Malcolm, 1985; & 58 Onthophagus gulo Arrow & M.P. & Arrow, 1931 \\
\hline
\end{tabular}




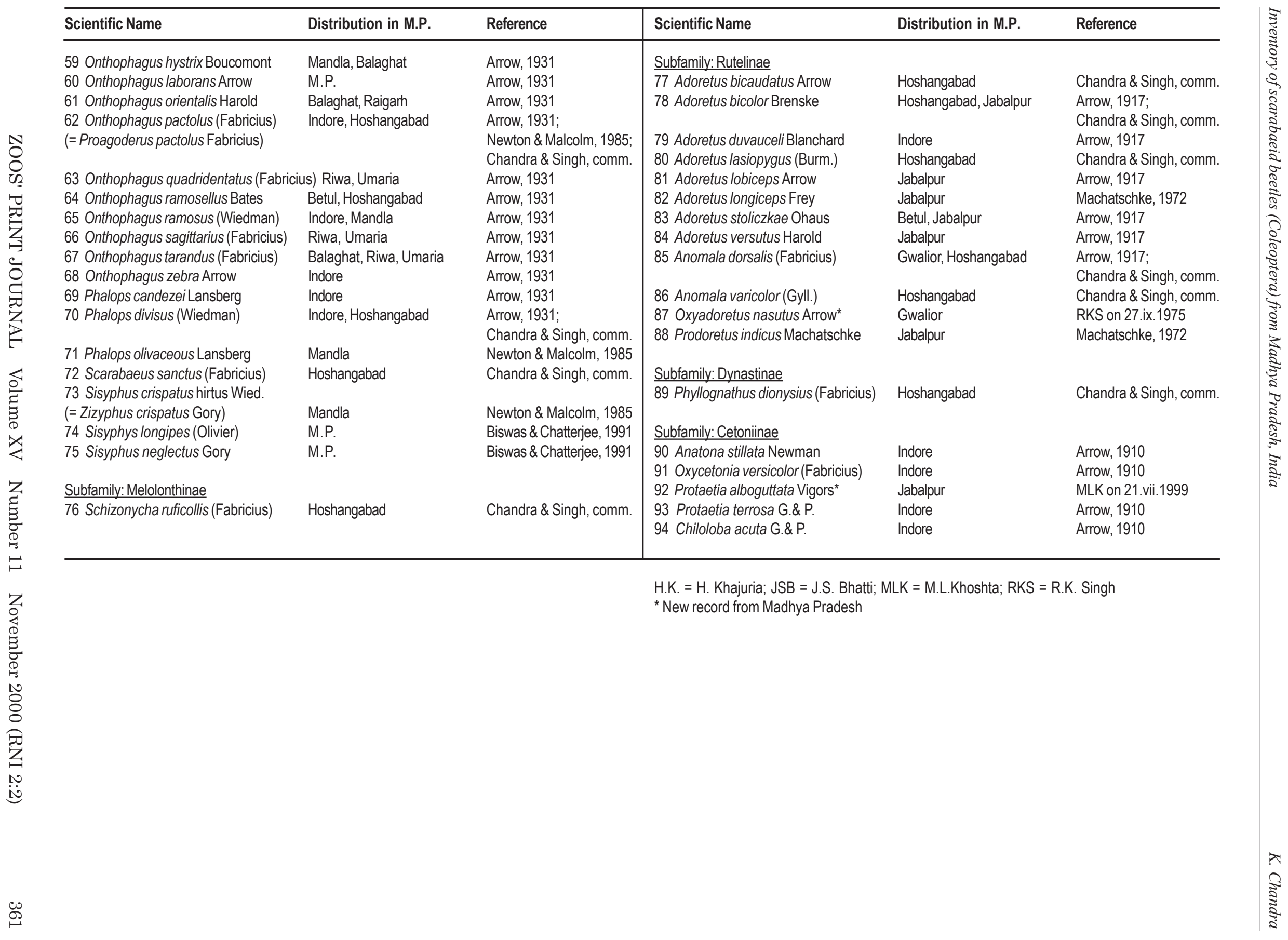


Biswas, S. and S.K. Chatterjee (1991). Insecta: Coleoptera: Scarabaeidae, State Fauna Series 1: Fauna of Orissa. Zoological Survey of India (Part 3): 243-262.

Chandra, K. and R.K. Singh (Communicated). On a collection of scarabaeid beetles from Pachmarhi Biosphere Reserve (Hoshangabad: Madhya Pradesh). Rec. Zool. Surv. India.

Dalla Torre, K.W. (1912-13). Coleopterrourum Catalogus (W. Junk). Scarabaeidae, Melolonthinae, pars 45, 47, 49, 50.

Fincher, G.T. (1981). The potential value of dung beetles in pasture system. J. Ga Ent. Soc. 16(2): 316-333.

Kuijten, P.J. (1983). Revision of the genus Hybosorus Macleay (Coleoptera: Scarabaeidaae: Hybosorinae). Zool. Verh. Leiden, 203: 149.

Machastschke, J.W. (1972). Coleopterorum Catalogus, (W. Junk), Scarabaeoidea, Melolonthidae, Rutelinae.

Newton, P.N. and J.C. Malcolm (1985). Dung and dung beetles in Kanha Tiger Reserve, Central Indian Highlands. J. Bombay nat. Hist. Soc. 85: 218-220.

NOTE ZOOS' PRINT JOURNAL 15(11): 362

\section{LARGE CONGREGATION OF ROOSTING HARRIERS IN SECUNDERABAD, ANDHRA PRADESH}

\section{Bhargavi Srinivasulu ${ }^{1}$ and C. Srinivasulu ${ }^{2}$}

${ }^{1}$ Senior Research Fellow, ${ }^{2}$ Senior Research Fellow (Wildlife) Wildlife Biology Section, Department of Zoology, Osmania University, Hyderabad, Andhra Pradesh 500007, India

${ }^{1}$ E-mail: bhargavi.srinu@usa.net.

${ }^{2}$ E-mail:masawa@satyam.net.in

On 17 December 1999, at about $1730 \mathrm{hr}$, while observing roosting harriers in a grassland in northern Secunderabad ( $c^{\prime} 17^{0} 26^{\prime}$ $17^{\circ} 27^{\prime} \mathrm{N} \& 78^{0} 27^{\prime}-78^{\circ} 28^{\prime} \mathrm{E}$ ) we noted large congregation of 570730 individuals of four species of harriers (Table 1) in 18 roosting spots. During our regular observation spanning seven years (1993-94 to 1999-2000) such a large congregation was seldom recorded. At the most 200-300 roosting individuals were noted. Altogether, five species of harriers, namely - Eurasian Marsh Harrier (Circus aeruginosus), Montagu's Harrier (Circus pygargus), Pallid Harrier (Circus macrourus), Pied Harrier (Circus melanoleucos) and Hen Harrier (Circus cyaneus) have been recorded roosting here (pers. observ.; Kannaiah \& Ganesh, 1995). The last species was the rarest, while Kannaiah \& Ganesh (1995) reported Pied Harrier to be the rarest during their observation.

The harriers displayed the pre-roost behaviour of flying over the roosting site just before or after sunset (Satheesan \& Rao 1990; Ganesh \& Kannaiah 1991). Most of the harriers roosted on the ground in tall grass $(>100 \mathrm{~cm})$, while some roosting spots were in areas with short grass $\left(c^{\prime} 40-60 \mathrm{~cm}\right)$ and on bare patches too.

Rahmani and Manakadan (1986) reported a large roost of harriers near Rollapadu comprising an estimated 800-1000 individuals. The large numbers of harriers indicate that the grassland in northern Secunderabad is an important assembly site for roosting and gathering of harriers over a few days before they finally depart on their migratory journey.

\section{Acknowledgements}

We thank Dr. V. Nagulu, and the Head, Department of Zoology for encouragement and facilities. We also acknowledge the Senior Research Fellowships granted to us by CSIR, New Delhi.

\section{References}

Ganesh, T. and P. Kannaiah (1991). Calls of harriers (Circus spp.) near Hyderabad, Andhra Pradesh. J. Bombay nat. Hist. Soc. 88(3): 448.

Kannaiah, P. and T. Ganesh (1995). Importance of kanchas in bird conservation. Mayura 12: 74-81.

Rahmani, A.R.and R. Manakadan (1986). A large roost of harriers in Andhra Pradesh, India. J. Bombay nat. Hist. Soc. 83(Suppl.): 203204.

Satheesan, S.M. and P. Rao (1990). Roosting and feeding of harriers in Secunderabad, Andhra Pradesh. J. Bombay nat. Hist. Soc. 87(1): 143.

Table 1. Estimated numbers of individuals of harriers observed roosting in a grassland in northern Secunderabad on 17 December 1999.

\begin{tabular}{|c|c|c|}
\hline \multirow[t]{2}{*}{ Species } & \multicolumn{2}{|c|}{ Estimated number of individuals } \\
\hline & Minimum & Maximum \\
\hline Pallid Harrier (Circus macrurus) & 220 & 280 \\
\hline Montagu's Harrier (Circus pygargus) & 180 & 250 \\
\hline Pied Harrier (Circus melanoleucos) & 100 & 120 \\
\hline Eurasian Marsh Harrier (Circus aeruginosus) & 70 & 80 \\
\hline Total & 570 & 730 \\
\hline
\end{tabular}

